\title{
Adolescentes Inseridos em um CAPSi: Alcances e Limites deste Dispositivo na Saúde Mental Infantojuvenil
}

\author{
Amanda Dourado Souza Akahosi Fernandes ${ }^{1}$ \\ Programa de Pós-Graduação em Terapia Ocupacional da Universidade Federal \\ de São Carlos, São Carlos, SP, Brasil \\ Thelma Simões Matsukura \\ Departamento de Fisioterapia e Terapia Ocupacional da Universidade Federal \\ de São Carlos, São Carlos, SP, Brasil
}

\begin{abstract}
Resumo
O objetivo do presente estudo foi identificar o dia a dia de adolescentes em sofrimento psíquico inseridos em um Centro de Atenção Psicossocial infantojuvenil (CAPSi) e compreender os alcances e limites deste dispositivo de cuidado na saúde mental infantojuvenil. Trata-se de um estudo de caso qualitativo, que envolveu a metodologia de Inserção Ecológica, fundamentada na teoria Bioecológica do Desenvolvimento Humano de Urie Bronfenbrenner. Participaram do estudo 13 adolescentes, com idades entre 12 a 18 anos, inseridos em um CAPSi de uma cidade de grande porte do estado de São Paulo. Os instrumentos utilizados para a coleta de dados foram o diário de campo e um formulário para identificação dos participantes. Para análise de dados do diário de campo, a pesquisadora realizou repetidas leituras e identificou extratos representativos da realidade observada, analisando-os descritiva e expressivamente. Foi possível identificar que as atividades realizadas no CAPSi são consideradas potencializadoras dos processos de inclusão social e do estabelecimento de relações sociais dos adolescentes. Evidenciaram-se algumas barreiras que tangem à dificuldade no trabalho intersetorial. Discutiu-se a potência das ações, vivências e oportunidades presentes no CAPSi e, ao mesmo tempo, os limites e avanços necessários no processo de inserção social.
\end{abstract}

Palavras-chave: Adolescente, saúde mental, serviços de saúde mental.

\section{Adolescents Treated in CPACA: Reach and Limitations of this Service for the Mental Health of Children and Adolescents}

\begin{abstract}
The objective of the present study was to identify the day-by-day of adolescents in psychic suffering that attend a Center of Psychosocial Attention for Children and Adolescents (CAPSi) and to understand what the reach and limitations of this service are within the care and mental health treatment of children and adolescents. This is a qualitative case study, that involved the methodology of Ecological Insertion, grounded on the Bioecological Theory of Human Development by Urie Bronfenbrenner. Thirteen adolescents treated in a CAPSi of a large city from the State of São Paulo and aged between 12 and 18 years
\end{abstract}

Endereço para correspondência: Universidade Federal de São Carlos, Centro de Ciências Biológicas e da Saúde, Departamento de Terapia Ocupacional, Rodovia Washington Luís, km 235 - SP-310, Monjolinho, São Carlos, SP, Brasil 13565-905. Fone: (16) 3351-8756. E-mail: amanda.d.fernandes@hotmail.com e thelma@ ufscar.br.

Financiamento: Fundação de Amparo à Pesquisa do Estado de São Paulo (FAPESP). 
old took part in the study. The instruments used for data collection were a diary and a form for the identification of participants. For the data analysis of the diary, the researcher read it repeatedly and identified representative fractions of the reality observed, analysing them descriptively and expressively. We identified that the activities in CAPSi are believed to enhance the processes of social inclusion and the establishment of social relations of adolescents. Some barriers that hinder intersectoral work have also been identified. We discussed the power of actions, experiences and opportunities at the CAPSi, but also, the limitations and necessary advances in the process of social inclusion.

Keywords: Adolescent, mental health, mental health services.

\section{Adolescentes Inserta en un CAPSI: Alcances y Límites de este Dispositivo en Mentales Salud Infantil y Juvenil}

\section{Resumen}

El objetivo de este estudio fue conocer como es el cotidiano de los adolescentes en sufrimiento psíquico que frecuentan un Centro de Atención Psicosocial infantojuvenil (CAPSi) y comprender los alcances y límites del espacio de cuidado en la salud mental de estos jóvenes y niños. Es un estudio de caso cualitativo, que involucró la metodología de Inserción Ecológica, basada en la teoría Bioecológica del Desarrollo Humano, de Urie Bronfenbrenner. Formaron parte del estudio 13 adolescentes, con edades entre 12 a 18 años, inseridos en un CAPSi de una ciudad grande del estado de São Paulo. Los instrumentos de recolecciónde datos han sido un diario de campo y un formulario de identificación de los participantes. Para analizar los datos del diario, la investigadora hizo repetidas lecturas e identificó fragmentos representativos de la realidad observada, los analizó descriptiva y expresivamente. Se pudo identificar que las actividades desarrolladas por el CAPSi son potenciadoras de los procesos inclusivos socialmente y de establecimiento de relaciones sociales de los adolescentes. Se encontraron evidencias de que hay algunas barreras que dificultan el trabajo de colaboración intersectorial. Se discutió la potencialidad de las acciones, vivencias y oportunidades del CAPSi y, simultáneamente, los límites y avances necesarios para el proceso de inserción social.

Palabras clave: Adolescentes, salud mental, servicios de salud mental.

No campo da saúde mental, a assistência à infância e adolescência foi marcada por estratégias de segregação e permaneceu em segundo plano, se comparada aos projetos referentes à assistência voltada a adultos (Guerra, 2003). A história das políticas destinadas aos direitos de crianças e adolescentes está envolta pelo controle do Estado sobre estes indivíduos e pela construção de um modelo de assistência centrado na institucionalização, visando garantir a proteção social (Ministério da Saúde, 2014).

Em 2004, o Ministério da Saúde brasileiro, com apoio de diversos setores da sociedade, governamentais ou não, instituiu o Fórum de Saúde Mental Infantojuvenil, criado com a finalidade de construir, coletiva e intersetorialmente, as bases, princípios e diretrizes de uma política pública de saúde mental dirigida a este seguimento (Ministério da Saúde, 2012). Observa-se que, nesta nova fase de ampliação das redes de cuidado à saúde mental infantojuvenil e de efetivação da nova política de saúde mental, os Centros de Atenção Psicossociais (CAPS) são serviços estratégicos para agenciar e ampliar as ações na área da saúde mental e as dos efeitos do uso de álcool e outras drogas. O serviço deve ser uma porta aberta às demandas de saúde mental de seu território e deve identificar populações específicas e vulneráveis que podem ser objeto de estratégias diferenciadas de cuidado (Amstalden, Hoffmann, \& Monteiro, 2010; Ministério da Saúde, 2014).

Para a população infantojuvenil, existem os Centros de Atenção Psicossocial Infantojuve- 
nis (CAPSi), especializados no atendimento de crianças e adolescentes com graves comprometimentos psíquicos. São equipamentos necessários para dar resposta à demanda em saúde mental em municípios com mais de 150 mil habitantes (Ministério da Saúde, 2011).

A partir das iniciativas existentes para implementação de uma política de saúde mental infantojuvenil, Delfini (2010) aponta que, no CAPSi, é essencial o estabelecimento de parcerias com toda a rede de saúde, além de setores como educação, lazer e justiça social para que seja possível ampliar as possibilidades de êxito no cuidado aos jovens. Apesar da proximidade com os demais CAPS, há a necessidade de um olhar diferenciado para esta população devido à fase de desenvolvimento em que se encontram, exigindo-se mais atenção no que se refere à inserção social em diversos ambientes, especialmente a escola (Delfini, 2010).

Mais recentemente, em 2011, o Ministério da Saúde institui por meio da portaria $\mathrm{n}^{\mathrm{o}} 3.088$, a Rede de Atenção Psicossocial (RAPS) que tem como finalidade a criação, ampliação e articulação de pontos de atenção à saúde para pessoas em sofrimento psíquico ou transtorno mental e com necessidades decorrentes do uso de álcool e outras drogas, no âmbito do Sistema Único de Saúde. Aponta-se a importância da RAPS para a infância e adolescência uma vez que ela enfatiza o cuidado a grupos mais vulneráveis.

Em consonância com as diretrizes da RAPS, em 2014, o Ministério da Saúde lançou o documento intitulado Atenção Psicossocial a Crianças e Adolescentes no SUS [Sistema Único de Saúde]: Tecendo Redes para Garantir o Cuidado. Este documento enfatiza a construção do cuidado em saúde mental infantojuvenil fundamentada em algumas diretrizes: reconhecimento da criança e adolescentes como sujeitos de direitos; acolhimento universal; encaminhamento implicado e corresponsável; construção permanente da rede e da intersetorialidade; trabalho no território; avaliação das demandas e construção compartilhada das necessidades de saúde mental.

Desta forma, o CAPS, ainda que sob perspectiva mais ampla e não centralizadora, perma- nece como importante equipamento especializado que compõe esta rede, sendo responsável por articular, além do cuidado clínico, os programas de reabilitação psicossocial.

Estudos envolvendo os CAPSi têm sido realizados sob diferentes enfoques e a indicação sobre a necessidade de aprofundar a compreensão e o conhecimento envolvendo estes dispositivos é unanime entre os pesquisadores (Beltrame, 2010; Delfini, Dombi-Barbosa, da Fonseca, Tavares, \& Reis, 2009; Falavina \& Cerqueira, 2008; Hoffmann, Santos, \& Mota, 2008; Oliveira, 2010).

Em relação ao sofrimento psíquico na adolescência, alguns estudos nacionais têm buscado identificar a prevalência de transtornos neste grupo. O estudo de Paula et al. (2008) objetivou analisar a prevalência e os fatores de risco e proteção para problemas de saúde mental entre adolescentes da sexta série de todas as escolas públicas e privadas de uma cidade do interior do estado de São Paulo. Os resultados apontaram que $12,5 \%$ apresentavam problemas de saúde mental.

Compreende-se a relevância da atenção à saúde mental neste período, à medida que intervenções voltadas para esses indivíduos pode ser fator decisivo e de extrema importância para a vida adulta, visto que esta fase é um período de desenvolvimento e de construção de projetos (Mângia, Assumpção, Quinta, \& Rufino, 2003).

É reconhecido que na adolescência algumas questões de saúde são intensificadas como, por exemplo, a depressão, surtos psicóticos, uso de substâncias psicoativas, o que implica na relevância de que o cuidado oferecido a esses indivíduos deve ser proposto diferentemente daqueles que se constroem para as crianças, que na maioria das vezes estão inseridas no mesmo serviço (Taño, 2014). Entretanto, nas produções e estudos do campo de saúde mental infantojuvenil, ainda são poucos os trabalhos que fazem a distinção entre essas duas fases do desenvolvimento, o que pode expressar a dificuldade de se pensar, propor e realizar ações específicas a esses indivíduos (Benetti, Ramires, Schneider, Rodrigues, \& Tremarin, 2007; Mângia et al., 2003; Santos, 2006). 
Nesta direção, este estudo focaliza adolescentes em sofrimento psíquico inseridos em um CAPSi, a partir da Teoria Bioecológica de Urie Bronfenbrenner, visto que esta viabiliza maior compreensão sobre essa etapa do desenvolvimento, com a especificidade do sofrimento psíquico. Destaca-se que, de acordo com o Modelo Bioecológico do Desenvolvimento Humano, diversos níveis de sistemas interagem de forma complexa e dinâmica e podem exercer influência sobre o desenvolvimento humano ao longo do curso de vida (Bronfenbrenner, 1996). Assim, o Modelo propõe que o desenvolvimento seja estudado por meio da interação sinérgica de quatro núcleos inter-relacionados: processo, pessoa, contexto e o tempo (Dell'Aglio, 2011).

\section{Objetivos}

O objetivo do presente estudo foi identificar o dia a dia de adolescentes em sofrimento psíquico inseridos em um CAPSi e compreender os alcances e limites desse dispositivo de cuidado na saúde mental infantojuvenil.

\section{Método}

Trata-se de um estudo de caso qualitativo que utilizou a metodologia de Inserção Ecológica.

\section{Inserção Ecológica}

A metodologia de Inserção Ecológica foi desenvolvida sobre as bases da Teoria Bioecológica do Desenvolvimento Humano, proposta por Urie Bronfenbrenner (Cecconello \& Koller, 2003). Tal metodologia privilegia a inserção dos pesquisadores no ambiente de pesquisa, com o objetivo de estabelecer proximidade com o seu objeto de estudo e prevê a sistematização dos quatro núcleos propostos por Bronfenbrenner, sendo eles: processo, pessoa, contexto e tempo (Paludo \& Koller, 2011).

Destaca-se que a base da investigação que adota a Inserção Ecológica ocorre a partir da interação recíproca, complexa e regular entre pesquisadores, participantes, objetos e símbolos presentes no contexto (Eschiletti-Prati, Couto, Moura, Poletto, \& Koller, 2008).

\section{Participantes}

Para o presente estudo, participaram 13 adolescentes inseridos no CAPSi, com idades entre 12 e 18 anos, que tiveram acompanhamento direto da pesquisadora durante sua a inserção no serviço. Aponta-se, porém, que, durante a Inserção Ecológica, diferentes atores vinculados ao cotidiano desses adolescentes estiveram presentes, como, por exemplo, os demais usuários do serviço (crianças e adolescentes), familiares e profissionais.

Quanto aos critérios de seleção adotados para a composição da amostra, além da idade dos participantes - que deveria ser de no mínimo 12 e no máximo 18 anos, os adolescentes deveriam estar inseridos no CAPSi há pelo menos três meses, não terem, como indicação de queixa principal, o uso abusivo de substâncias psicoativas e terem condições de compreender e manter um diálogo, segundo avaliação dos profissionais do serviço.

$\mathrm{Na}$ Tabela 1 a seguir apresentam-se as informações referentes aos adolescentes participantes do estudo:

\section{Tabela 1}

Caracterização dos Adolescentes da Pesquisa

\begin{tabular}{ccc}
\hline Variável & Classificação & Participantes \\
\hline \multirow{2}{*}{ Sexo } & Masculino & 8 \\
& Feminino & 5 \\
Idade & 12 a 14 anos & 7 \\
& 15 a 18 anos & 6 \\
Frequenta & Sim & 6 \\
a Escola & Não & 7 \\
& Fundamental 1 & 2 \\
Escolaridade & Fundamental 2 & 8 \\
& Ensino médio & 3 \\
Repetência & Sim & 7 \\
escolar & Não & 6 \\
& Mãe e irmãos & 2 \\
Composição & Pais e irmãos & 5 \\
familiar & Outros familiares & 6 \\
\hline
\end{tabular}


Observa-se, a partir das informações apresentadas na Tabela 1, que a maioria dos participantes são meninos (8); 7 adolescentes possuem entre 12 e 14 anos e 6 adolescentes encontram-se na faixa etária entre 15 e 18 anos.

Em relação à escolaridade a maioria cursa ou parou os estudos no Ensino Fundamental II. Dos 13 adolescentes, 7 não frequentam a escola, 5 dos quais também informaram repetência. Apenas 5 dos adolescentes moram com ambos pais, 2 moram com as mães e os demais residem com os avós e/ou outros familiares.

Além disso, outras informações clínicas foram coletadas durante a inserção ecológica. A maioria dos adolescentes participantes deste estudo apresentou problemas desde a infância (8) e apenas um, dos 4 jovens que tiveram o início dos problemas na adolescência, não passou por internação psiquiátrica. Quanto às modalidades de inserção no CAPSi, a minoria (4) se encontra na modalidade não intensiva. Em relação aos tipos de atividades que os adolescentes participam no CAPSi, 11 adolescentes frequentam mais que uma atividade e o atendimento individual é oferecido para 10 dos 13 adolescentes.

Em relação às queixas ou diagnóstico identificado em prontuários ou relatados pela equipe, há referencias a quadros psicóticos/ esquizofrenia, isolamento e dificuldade de relacionamento social, quadros depressivos, pensamento desorganizado, deficiência mental, problemas de comportamento, hiperatividade e autismo.

\section{Contexto da Pesquisa: CAPS Infantojuvenil}

O estudo foi realizado em um serviço público de saúde mental destinado à clientela de crianças e adolescentes - CAPSi - localizado em um município de grande porte do estado de São Paulo.

Havia um total de 1346 prontuários registrados até dezembro de 2012 e destes, 162 usuários estavam em tratamento. Dos 162 usuários, 99 eram adolescentes, sendo 67 meninos e 32 meninas.

O CAPSi contava com uma equipe técnica de 23 profissionais, composta por médicos, psi- cólogos, terapeutas ocupacionais, educadores sociais, enfermeiros e assistente social. Havia também profissionais ligados à administração e manutenção da unidade, como auxiliares administrativos, motorista, pessoal de limpeza e segurança.

\section{Instrumentos para Coleta de Dados}

Formulário de Identificação. Foi utilizado um formulário de identificação para registro de dados pessoais e clínicos dos adolescentes, composto por 19 questões que buscaram caracterizar os participantes-foco. As informações registradas no Formulário foram obtidas a partir dos adolescentes, profissionais do serviço e prontuário.

Diário de Campo. Foi utilizado um diário de campo no qual foram feitos os registros sistemáticos dos dados obtidos durante a Inserção Ecológica. No diário estão contidas descrições, trechos de fala dos participantes, impressões e sentimentos da pesquisadora.

O uso do diário de campo no procedimento de Inserção Ecológica, possibilita abordar de forma mais diretiva as categorias a serem utilizadas tanto no momento descritivo quanto reflexivo. Assim, os registros no diário, orientados pela Teoria Bioecológica, retratam o olhar do pesquisador sobre os pontos essenciais do modelo, tais como as características e atributos dos participantes; os contextos dos quais participam; as atividades compartilhadas; os papéis desempenhados e o estabelecimento das relações, perpassando por esses elementos o tempo, no qual as relações e as atividades passam a ser compreendidos (Afonso, Silva, Pontes, \& Koller, 2015).

\section{Procedimentos}

Conforme a proposta de Inserção Ecológica, o processo foi previamente esclarecido, planejado e combinado com o serviço, com os profissionais, adolescentes e seus familiares e ocorreu durante um período de cinco meses (entre dezembro de 2012 e abril de 2013).

Anteriormente à coleta de dados, foram realizados contatos com o CAPSi explicando a pesquisa e identificando o melhor período para sua realização. Conforme os objetivos da pesquisa 
e o contexto de inserção, definiu-se, juntamente com o serviço, que a entrada da pesquisadora no contexto se iniciaria no período de férias, para que fosse possível começar a aproximação com o ambiente, com os profissionais e alguns adolescentes que circulassem neste período. Os três primeiros meses de inserção coincidiram com o período de festas e férias escolares e foi possível permanecer no serviço em torno de três vezes por semana para acompanhar as atividades programadas para este período e realizar a aproximação com profissionais e usuários do centro, por meio de conversas informais e da participação da pesquisadora nas atividades. Desta forma, durante o período, atividades de culinária, oficinas e grupos foram acompanhados pela pesquisadora e os contatos com os adolescentes foram se efetivando.

Finalizadas as férias, foi possível aprofundar e/ou estabelecer novos vínculos com os adolescentes, famílias e profissionais, por meio da permanência diária da pesquisadora no CAPSi durante todos os dias da semana, sete horas por dia.

No $4^{\circ}$ mês de inserção, o convite para a participação dos adolescentes que respondiam aos critérios estabelecidos para o estudo foi formalizado e explicações sobre o mesmo foram apresentadas. A partir do interesse do adolescente, as respectivas famílias foram também formalmente contatadas.

A cada dia e cada encontro no CAPSi e em outros contextos, realizava-se o registro no diário. Foram nos momentos de convivência e nos grupos que a relação entre pesquisadora e participantes se intensificou e o cotidiano de cada adolescente emergiu. Observa-se que, apesar de muitos usuários do serviço não comporem a amostra de participantes, todos fizeram parte deste processo, devido à metodologia adotada e à inserção diária no campo.

A saída da pesquisadora do campo foi previamente combinada com o CAPSi. Desta forma, quando se considerou que o período de inserção havia permitido a compreensão da realidade investigada, a pesquisadora conversou com a equipe, fez uma devolutiva do processo de inserção e da pesquisa e iniciou a desvinculação com os usuários. Todos os usuários e familiares foram notificados a respeito da saída da pesquisadora, algumas despedidas nos grupos foram realizadas e, paulatinamente, a pesquisadora foi se retirando do campo.

\section{Análise de Dados}

Para a análise dos registros efetivados no diário de campo foi retomada a proposta metodológica de Inserção Ecológica, assim como os objetivos do estudo. Desta forma, após leitura repetida de todo o conteúdo do diário, foram selecionados extratos que contemplassem tanto os objetivos como o referencial teórico e a metodologia adotada. Estes extratos foram destacados e colocados para apresentação dos resultados em forma de narrativa-descritiva.

Yin (1994) aponta que a análise de dados, advindos de estudos de caso, pode estar baseada em proposições teóricas ou na descrição do caso. Desta forma, os dados coletados durante a Inserção Ecológica no CAPSi presentes no diário de campo foram analisados e apresentados descritivamente, por meio de alguns extratos retirados do diário.

\section{Resultados}

Os resultados a seguir serão apresentados a partir de extratos retirados do diário de campo, tendo como base os quatro núcleos propostos pela Teoria Bioecológica de Bronfenbrenner: processo, pessoa, contexto e o tempo.

\section{Processos Proximais - Os Adolescentes, as Atividades e o CAPSi}

Os processos proximais foram identificados em diferentes momentos da Inserção Ecológica, desde o engajamento da pesquisadora nas atividades oferecidas; na relação estabelecida entre os adolescentes: entre estes e os profissionais; assim como em relação às atividades, conforme verifica-se nos extratos do diário de campo a seguir: É muito rico observar quando os usuários
chegam ao CAPSi e logo correm para os
braços do guarda, por exemplo, cumpri-
mentam, abraçam e passam o horário de 
almoço conversando com ele. Assim como é muito rico ver as crianças pedirem para as auxiliares de limpeza que façam um agrado, como um bolo de aniversário, um docinho, um lanche fora de hora e depois agradecem com imenso carinho. É esse compartilhar de afetos, momentos de trocas que tornam esses profissionais parte da equipe e fundamentais para o CAPSi.

Observa-se no extrato acima o papel que essas relações estabelecidas e construídas no dia a dia do CAPSi, entre os usuários e profissionais, e a importância que elas possuem neste contexto. Importa destacar que esses processos ocorrem não só com os profissionais técnicos envolvidos diretamente com o cuidado aos usuários, como também com os profissionais de apoio ao serviço, que, indiretamente, passam a fazer parte do contexto de vida desses sujeitos.

Sobre a relação dos adolescentes com os usuários, observou-se que, muitas vezes, estas relações envolveram o companheirismo, o afeto, e a compreensão. Da mesma forma, foi possível observar a relação estabelecida entre os adolescentes e as atividades propostas, a importância destas no cotidiano dos mesmos, no tratamento e nas tentativas de inclusão destes jovens em outros contextos externos ao CAPSi:

É interessante observar a dinâmica do grupo de esporte, pois diferentes processos acontecem. L é um dos primeiros a ser escolhido no time e durante o jogo é possivel observar maior interação dele com os usuários, pois, quando faz gols, além de sorrir e comemorar timidamente, os outros correm para cumprimentá-lo, abraçá-lo e ele discretamente retribui. Além do L, temos o K, uma criança que se angustia e fica extremamente nervosa quando está perdendo. Em seus momentos de nervosismo, há um processo dos membros do seu time de acalmá-lo, de explicar o que aconteceu e compreender as angústias dele. Em alguns momentos em que ele se irritou muito, o jogo foi paralisado e realizou-se uma roda de conversa. Nessa roda alguns combinados foram reafirmados e no próximo encontro tudo aconteceu bem, de acordo com os combinados.
Como fator imprescindível no processo de Inserção Ecológica, aponta-se que - por meio das vivências com os adolescentes, das conversas informais e do compartilhamento de um mesmo espaço-tempo - foi possível apreender o dia a dia dos adolescentes e também fazer parte dele. Foi essa relação que possibilitou que a pesquisadora adentrasse no contexto do CAPSi e, consequentemente, fizesse parte da dinâmica do serviço.

A maioria dos adolescentes foi muito receptiva, se aproximaram de mim com seu jeito peculiar e, apesar das dificuldades, interagiam. Com o tempo, a minha aproximação com eles tornou-se grande, de forma que ficávamos juntos conversando, em outros ambientes, na convivência.

Outro tipo de atividade que participei com os adolescentes foi em atendimentos individuais. Em um determinado momento eu estava na convivência brincando de bola com um adolescente e a psiquiatra o chamou para sua primeira consulta. $O$ adolescente ficou inseguro e disse à psiquiatra que só entraria na sala se eu pudesse estar junto. Prontamente ela disse que não havia problema algum da minha presença. Nós entramos e a psiquiatra iniciou o atendimento.

\section{Núcleo Pessoa - Os Adolescentes Inseridos no CAPSi}

A inserção da pesquisadora permitiu conhecer cada adolescente, suas individualidades, potencialidades, características, desejos e planos futuros. Por meio desse reconhecimento, foi possível compreender, sobre os processos internos, o sofrimento psíquico e a sua relação com o dia a dia dos adolescentes.

"Conversando com alguns adolescentes sobre suas qualidades, um deles, com todo o comprometimento revelou - 'Tudo é porcaria isso daqui' [se referindo a ele mesmo]. Já outro jovem, apontou como qualidade o fato de ser amoroso".

Em diversas ocasiões, os adolescentes, familiares e profissionais falaram sobre as dificuldades dos usuários de estarem inseridos em outros contextos, a dificuldade de fazerem amigos 
e em que aspectos a doença mental mudou em suas vidas.

Em um momento de conversa na convivência uma das adolescentes afirma o desejo de poder sair, passear, porém há alguns impeditivos, como o fato dos pais não permitirem por achar que ela não ficará bem ou por não confiarem nela. A adolescente relatou que é muito difícil ir à casa de amigas, ou passear no campo, sendo que tem ocasiões que não aguenta e acaba fugindo.

\section{Núcleo Contexto - CAPSi, Família e Escola}

Quanto ao núcleo contexto, a pesquisadora pôde conhecer não só o contexto do CAPSi onde esteve inserida diariamente, como também o contexto familiar e escolar, por meio dos relatos dos adolescentes e da própria família.

Ressalta-se que muitas limitações no contexto familiar surgiram, como, por exemplo, as dificuldades familiares, as fragmentações e os conflitos.

Quanto ao contexto familiar de um dos adolescentes participantes, o técnico da equipe relata que os membros da família não compreendem o que ele tem, do que ele sofre, então muitas vezes acham que tem que dar um tapa, pôr de castigo e isso acaba isolando cada dia mais o adolescente da familia.

$\mathrm{Na}$ escola, os relatos expressam as dificuldades de aprendizagem, de inclusão e de relações sociais, como ilustram os trechos do diário de campo, a seguir.

Quanto ao contexto escolar, o técnico da equipe afirma ser bem prejudicado para o adolescente A. Por ser um menino muito passivo sempre teve muita dificuldade na escola, de relação mesmo com outros meninos... então ele não frequenta escola, não consegue frequentar, justamente pela questão de estar em grupo, das relações sociais, das exigências, ele tem muita vontade de aprender, mas não consegue estar em um grupo escolar tradicional.

Em uma conversa sobre a escola, " $F$ " me contou sobre sua experiência: "Eu faço besteira, de primeiro eu jogava cadeira, mesa na escola, jogava um monte de cadeira, só ouvia barulho... agora eu só jogo às vezes quando eu fico muito nervoso. Fica tudo no chão as cadeiras".

Já sobre o microssistema do CAPSi, verificou-se que a maioria dos adolescentes permanecem vários dias da semana neste contexto, ainda que fosse esperado e desejado que outros microssitemas pudessem ser acessados por eles, como pode ser verificado nos extratos a seguir.

"O adolescente relatou que é o pai que leva pro CAPS todos os dias, depois fica até cinco horas. Ele fica fazendo algumas coisas, brincando, cantando... Às vezes ele fica sozinho sem fazer nada, escutando música, ou assistindo TV..."

“... Outro aponta que, quando não está no CAPSi, fica em casa, e dorme o dia inteiro, só dorme".

Sobre a avaliação dos técnicos, acerca do alcance e limites das ações de cuidado e atenção oferecidas pelos dispositivos de atenção à saúde mental infantojuvenil, encontram-se, a seguir, as considerações apresentadas à pesquisadora durante as conversas no campo.

... [eu acho que] responde com todas as dificuldades e limitações, mas eu acho que o CAPSi procura e desenvolve hoje projetos terapêuticos dentro da unidade em consonância com as diretrizes da política macro. É aquilo, ela está muito bonita no papel, mas a gente tem muito aqui no dia a dia, no cotidiano, a gente esbarra às vezes com uma série de limitações, seja material, transporte, conciliar atendimento, clínica, o tratamento, com outras atribuições, como matriciar lá fora. Então eu acho que a gente está caminhando nisso, mas eu acredito que a gente faça a política, com muitas limitações do município em se adequar.

O CAPSi é um importante recurso para tratamento dos usuários, apesar de muitas vezes se ver limitado por barreiras, sejam estas institucionais ou de outra natureza Além disso, o relato dos usuários é que o CAPSi tem ajudado na melhora dos sintomas, na inclusão social, e como importante fonte de suporte social. 


\section{Núcleo Tempo- Perspectivas Futuras}

Por fim, o núcleo tempo. O tempo permite examinar a influência sobre o desenvolvimento humano de mudanças e continuidades que ocorrem ao longo do ciclo de vida. Este elemento é analisado em três níveis - micro, meso e macrotempo.

Durante a inserção de cinco meses no serviço, a pesquisadora pôde acompanhar mudanças, transformações e continuidades, assim como conhecer um pouco de cada um e suas historias de vida.

Durante o tempo que passava na convivência, tive muito contato com os pais e avós dos usuários. Uns liam revistas, outros tricotavam, outros conversavam o tempo todo, "trocando figurinhas". Assim, pude conhecer suas vidas, seus cotidianos, dificuldades, suas histórias. Muitas historias são marcadas por sofrimento familiar, como drogas, conflitos, abandono, prostituição, doenças e, outras, mesmo com as dificuldades, há a esperança, a confiança e a fé de um futuro melhor.

Em relação ao microtempo, foi possível analisar a continuidade e descontinuidade, observadas dentro dos pequenos episódios dos processos proximais por meio da interação entre os usuários, serviço e profissionais, conforme relatado no diário de campo:

Contando um pouco sobre a relação com a família, a adolescente $C$, relata ter uma boa relação com a mãe - "Quando a minha mãe vai lá, a gente brinca, vai visita minha tia, sai, conversa, passeia, a gente vai no shopping, vai no bosque, na casa dos parentes e amigos".

Em outro momento de conversa com as famílias, uma delas relatou que a relação com o adolescente varia entre amor e ódio, de forma que quando estão de acordo tudo fica tranquilo, e quando não eles brigam. Ultimamente o familiar relata não estar se entendendo com o adolescente, porém de acordo com o mesmo de 1 a 100 é $20 \%$... que ele é assim violento, de bater boca, de agressão física.
Quanto ao mesotempo, foi possível conhecer e analisar as mudanças e transformações em dias e semanas, como a chegada de novos usuários, a inserção de alguns nas atividades, os relatos de melhora, assim como a piora com as consequentes internações.

Pude também acompanhar a internação de dois adolescentes. Antes das internações me recordo dos momentos em que eles estavam bem, participando de diferentes atividades, até que em algumas semanas as vozes começaram a surgir, os delírios persecutórios sempre presentes e com isso o distanciamento da realidade, as dificuldades de se relacionar, interagir e estar presente nas atividades. Em uma das internações estive presente até o momento em que o adolescente entrou na van, acreditando que ia dar um passeio... Pouco mais de uma semana ele retornou ao CAPSi e apesar do comportamento menos agitado e a consciência de que tivera uma crise, alguns delírios permaneciam.

O macrotempo esteve presente nos relatos referentes às perspectivas e planos para o futuro, o desejo da profissionalização, casamento, constituição de família, assim, como a historia intergeracional das famílias, permeada nos relatos dos profissionais e familiares.

A relação da família é bem delicada. Me recordo um pouco sobre a história e alguns fatos onde o padrasto tentou matar essa irmã, teve um surto, achando que tinha que sacrificar aquela criança para adorar a Deus, uma familia muito estranha, com histórias muito complicadas. A história da mãe dele também afeta muito a familia, ele é meio que tachado pela história da vida da mãe dele, isso eu acho que é bem difícil.

\section{Discussão}

Observa-se que através da metodologia de Inserção Ecológica foi possível conhecer com profundidade o dia a dia dos adolescentes inseridos no CAPSi, assim como os diferentes contextos nos quais estes adolescentes estão inseridos como a escola, o CAPSi e a família. 
Quanto aos objetivos do CAPSi, verificou-se que estes englobam o atendimento à população, o acompanhamento clínico e a inserção social dos usuários, tendo como funções a atenção diária, o acolhimento, cuidado clínico, inserção social e tais objetivos e ações são coerentes com as políticas públicas que ressaltam o foco no sujeito, em suas singularidades, sua história, cultura e a vida cotidiana (Ministério da Saúde, 2012).

Da mesma forma, os resultados revelaram as ações que buscam responder às premissas da reabilitação psicossocial e das políticas públicas e também os desafios e limites que estão ali implicados.

Quanto às propostas de cuidado oferecidas no CAPSi, focalizado na presente pesquisa, há uma gama de atividades que visam a inserção dos usuários. Estas permeiam, desde a convivência, os atendimentos individuais, grupos e oficinas dentro do CAPSi, até intervenções externas que ocorrem em locais públicos do município ou na residência de alguns usuários.

Identificou-se neste estudo que tais práticas são realizadas na tentativa de que estes espaços se tornem dispositivos para que os processos de inclusão social e de estabelecimento de relações sejam possíveis. São momentos nos quais os adolescentes, por mais dificuldades que estejam vivenciando, são acolhidos, ouvidos, respeitados e os processos proximais são estabelecidos; assim como o núcleo relativo à pessoa se torna reconhecido, influenciando e sendo influenciado pelos processos proximais. Além disso, há trocas afetivas, compartilhamento de atividades, estabelecimento de laços, fortalecimento de suas redes.

Vale aqui destacar a importância do espaço de convivência/ambiência para o contexto do CAPSi, família e dos adolescentes. No CAPSi desta pesquisa, a convivência não é formalizada enquanto recurso terapêutico. No entanto, a gama de processos e relações que ocorrem neste espaço reafirmam considerações da literatura acerca da potencialidade da convivência nestes equipamentos (Moraes, Dias, Romio, \& Peixoto, 2011; Ronchi \& Avellar, 2013).
Compartilham-se aqui as considerações de Moraes et al. (2011) que destacam a importância deste recurso de ambiente dentro de um CAP$\mathrm{Si}$, visto que potencializa o trabalho dos profissionais no serviço, oferecendo o acolhimento, a produção de subjetividades, a escuta aos usuários e familiares. Observa-se que o conceito de ambiência se soma à proposta da clínica ampliada, pois o "ambiente" não se restringe ao espaço físico, mas ressoa no espaço social e de relações interpessoais.

Ainda, durante a inserção, revelou-se que muitos adolescentes só conseguem oportunidades de ação ou interação neste espaço de convivência, como, por exemplo, jogar bola, encontrar e conversar com outro usuário que frequenta o CAPSi no mesmo horário, dentre outras. Verificou-se que alguns processos proximais nem sempre são possíveis em outros contextos ou, mais grave, para boa parte dos usuários, este é um dos únicos contextos de relação, à medida que há a ausência de alternativas de pertencimento e/ou trânsito por outros espaços, como a escola, visto que alguns sujeitos não a frequentam. Assim, é no espaço da convivência do CAPSi que se inicia a construção de um dia a dia onde relações sociais são estabelecidas, vínculos e amizades surgem e há a possibilidade de compartilhar atividades.

Quanto ao microssistema da família, os resultados apontaram não só a presença de conflitos familiares e dificuldade de relacionamento, como também uma estrutura familiar muitas vezes desorganizada e conturbada, marcada pela violência, fragmentação e conflitos. Para a reflexão desses resultados, é pertinente utilizar os conceitos e estudos que tratam dos fatores e mecanismos de risco (Rutter, 1987). Assim, é possível que a violência, desestruturação familiar, dentre outros aspectos compreendidos como fatores de risco, podem implicar e/ou aumentar o sofrimento mental dos adolescentes. Pesquisas vêm indicando, por exemplo, a associação entre vivenciar violência e sofrer problemas de saúde mental ao longo do ciclo de crescimento e desenvolvimento humano (Assis, Avanci, \& Oliveira, 2009). 
Entretanto, importa refletir também sobre outro ângulo, visto que a presença de sofrimento mental na família pode vir a afetar todos seus membros. No estudo de Navarini e Hirdes (2008), os resultados apontam que as famílias que convivem com a doença mental possuem dificuldades de manejo em situações de crise, sentem culpa pela doença, solidão e desamparo. Quando aparecem os primeiros sintomas, os sentimentos como o medo, a tristeza, a vergonha e a raiva, assim como o afeto e o cuidado estão, ao mesmo tempo, presentes. Os autores destacam que a doença significa mais do que um conjunto de sintomas, ela possui outras representações de ordem simbólica, moral, social ou psicológica para o doente e sua família (Navarini \& Hirdes, 2008).

Assim, compreende-se que as rupturas existentes no contexto familiar desses adolescentes, assim como as dificuldades de relacionamento e a falta de suporte social oferecidos pelos familiares presentes no cotidiano desses jovens, influenciam no desenvolvimento dos adolescentes, como também são influenciados por eles.

Ainda no que tange aos contextos, aponta-se não só para a importância do reconhecimento dos microssistemas, mas também para o contexto macro, através do reconhecimento da necessidade de transformação e efetivação das políticas públicas a esse seguimento.

$\mathrm{Na}$ conjuntura do atual cenário das Políticas Públicas a este seguimento, torna-se fundamental pensar no cuidado a essa população a partir da construção da RAPS, sendo que essa rede é composta por equipamentos que permeiam desde a atenção básica, como a especializada e reabilitação psicossocial. O objetivo da RAPS é ampliar o acesso da população nos diferentes pontos de atenção e garantir a articulação e integração desses pontos de atenção das redes de saúde no território, qualificando o cuidado por meio do acolhimento, do acompanhamento contínuo e da atenção às urgências. A partir desta perspectiva, é possível considerar que, efetivar a ampliação da inserção destes adolescentes em outros microssistemas, seja mais viável.

Os resultados também revelam a necessidade de efetivação de estratégias e ações inter- setoriais de trabalho em rede que atendam às necessidades e demandas desta população, principalmente, no que tange a educação.

Em relação ao espaço escolar, evidenciou-se que, para além da desvinculação dos adolescentes da escola - realidade da maior parte dos jovens focalizados neste estudo -, tem-se também uma complexidade presente na situação dos adolescentes que a frequentam, o que reafirma a imprescindível necessidade de ações intersetoriais nesse campo (Fleitlich, 2005; Matsukura, Fernandes, \& Cid, 2012; Santos, 2006).

Alguns estudos apontam o impacto da saúde mental da criança e do adolescente e possibilidades de aprendizado e inserção no contexto escolar (Almeida-Filho, 1981; Santos, 2006). O estudo de Santos (2006) revelou que, dentre as queixas referentes aos adolescentes atendidos em um programa de psicologia ( $n=129), 30,2 \%$ apontam dificuldades de aprendizagem e 19,4\%, desinteresse pela escola e que a maioria dos adolescentes dessa pesquisa não frequentavam a escola. Assim, tanto os resultados obtidos nesta pesquisa, como os achados na literatura reforçam a emergência de ações articuladas entre os contextos. No entanto, tal articulação é ainda um grande desafio, como verificado neste estudo, a partir de relato de um profissional do CAPSi, as tentativas de parceria com o setor da educação se encontram bastantes limitadas.

Há que se ressaltar que a saúde mental da criança e do adolescente está fortemente relacionada ao contexto escolar, sendo fundamental refletir sobre as possibilidades de cuidado existentes para esta população, uma vez que as limitações observadas caminham em direção a um sistema educacional e social excludente.

Considera-se também que a doença mental e sua complexidade trazem consigo desafios e demandas com as quais a escola, os serviços de saúde, a assistência social e judiciária, assim como as pesquisas na área, ainda que adotado o paradigma da inclusão, não têm alcançado melhorias.

Compreende-se que o núcleo tempo está presente e articulado a todos os núcleos e coloca a dimensão do dia a dia, das transformações, das vidas e das ações em movimento. Colocar 
foco neste núcleo potencializa a concepção dos processos que ocorrem nos contextos e não só fornece a dimensão das transformações, mas também daquilo que não se alterou, indicando rotas para o investimento de ações e Políticas Públicas, por exemplo.

\section{Considerações Finais}

O objetivo do presente estudo foi compreender o dia a dia de adolescentes em sofrimento psíquico inseridos em um CAPSi e relacioná-los com os alcances e limites desse equipamento de saúde mental infantojuvenil.

A partir do objetivo e da utilização da metodologia de Inserção Ecológica, foi possível contemplar os quatro núcleos apontados por Bronfenbrenner presentes no processo de desenvolvimento humano: o processo proximal, a pessoa, o contexto e o tempo.

No dia a dia dos adolescentes, foi possível compreender os processos proximais estabelecidos nas relações familiares, nas amizades, com os profissionais do CAPSi, com as atividades e com a própria pesquisadora.

Quanto ao núcleo pessoa, a inserção da pesquisadora e as entrevistas permitiram identificar as potencialidades e dificuldades de cada adolescente, suas individualidades, características, desejos e planos futuros. Este reconhecimento individual permitiu melhor compreensão sobre os processos internos, o sofrimento psíquico intenso e a sua relação com o dia a dia.

$\mathrm{O}$ contexto esteve fortemente presente à medida que a pesquisadora esteve inserida no CAP$\mathrm{Si}$ diariamente, vivenciando o serviço. Além disso, foi possível participar de atividades externas, em outros contextos de inserção dos adolescentes, e ainda, indiretamente, por meio dos relatos dos participantes, profissionais e familiares, outros contextos foram descortinados, como o contexto familiar e escolar. Estudos futuros são necessários para o reconhecimento de outros contextos e aprofundamento sobre os mesmos.

Em relação ao tempo, durante os cinco meses de inserção, a pesquisadora vivenciou as transformações, o desenvolvimento e os processos ocorridos, tanto em relação ao micro como ao mesotempo. Neste período, presenciaram-se os processos proximais existentes, o desenvolvimento dos adolescentes, assim como o inicio de crises, internações e retornos, o processo de inserção dos adolescentes nos grupos, entre outras. Também foi possível identificar - por meio dos relatos - as histórias e transformações ocorridas entre as gerações.

Observa-se que, a partir dos resultados obtidos e da discussão apresentada, muitas questões surgiram, possibilitando pesquisas futuras. Dentre elas apontam-se os limites para a participação e acesso a outros microssistemas, em especial a escola. Nesta direção, estudos que abordem os processos que, de fato, viabilizem a inclusão social desta população e suas particularidades são fundamentais.

\section{Referências}

Afonso, T., Silva, S. S. C., Pontes, F. A. R., \& Koller, S. H. (2015). O uso do diário de campo na inserção ecológica. Psicologia \& Sociedade, 27(1), 131-141. doi:10.15448/1980-8623.2015.1

Almeida-Filho, N. (1981). Development and assesment of the QMIP: A Brazilian children's behavior questionnaire for completion by parents. Social Psychiatry, 16, 205-212.

Amstalden, A. L. F., Hoffmann, M. C. C. L., \& Monteiro, T. P. M. (2010). A política de saúde mental infantojuvenil: Seus percursos e desafios. In O. Y. Tanaka, Atenção em saúde mental para crianças e adolescentes no SUS. São Paulo, SP: Hucitec.

Assis, S. G., Avanci, J. Q., \& Oliveira, R. V. C. (2009). Socioeconomic inequalities and child mental health. Revista de Saúde Pública, 43(1), 92-100. doi:10.1590/S0034-89102009000800014

Beltrame, M. M. (2010). Infância e saúde mental: Reflexões sobre a dinâmica de trabalho de um CAPSi (Dissertação de mestrado, Universidade Estadual de Maringá, PR, Brasil). Recuperado em http://www.ppi.uem.br/Dissert/PPI-UEM 2010 Marina\%20Beltrame.pdf

Benetti, S. P. C., Ramires, V. R. R., Schneider, A. C., Rodrigues, A. P. G., \& Tremarin, D. (2007). Adolescência e saúde mental: Revisão de artigos brasileiros publicados em periódicos nacionais. Cadernos de Saúde Pública, 23(6), 1273-1282. doi:10.1590/S0102-311X2007000600003 
Bronfenbrenner, U. (1996). Ecologia do desenvolvimento humano: Experimentos naturais e planejados. Porto Alegre, RS: ArtMed.

Cecconello, A. M., \& Koller, S. H. (2003). Inserção ecológica na comunidade: Uma proposta metodológica para o estudo de famílias em situação de risco. Psicologia: Reflexão e Crítica, 16(3). doi:10.1590/S0102-79722003000300010

Delfini, P. S. de S. (2010). Centro de Atenção Psicossocial infantojuvenil e estratégia de saúde da família: Articulação das ações voltadas à saúde mental de crianças e adolescentes (Dissertação de mestrado, Faculdade de Saúde Pública, Universidade de São Paulo, SP, Brasil). Recuperado em http://www.teses.usp.br/teses/disponiveis/6/6136/tde-16122010-161656/pt-br.php

Delfini, P. S. de S., Dombi-Barbosa, C., da Fonseca, F. L., Tavares, C. M., \& Reis, A. O. A. (2009). Perfil dos usuários de um Centro de Atenção Psicossocial infantojuvenil da grande São Paulo. Revista Brasileira de Crescimento e Desenvolvimento Humano, 19(2), 226-236. doi:10.1590/ S0103-21002012000100009

Dell'Aglio, D. D. (2011). Prefácio. In S. H. Koller (Ed.), Ecologia do desenvolvimento humano: Pesquisa e intervenção no Brasil. São Paulo, SP: Casa do Psicólogo.

Eschiletti-Prati, L., Couto, M. C. P. P., Moura, A., Poletto, M., \& Koller, S. H. (2008). Revisando a inserção ecológica: Uma proposta de sistematização. Psicologia: Reflexão e Crítica, 21(1), 160169. doi:10.1590/S0102-79722008000100020

Falavina, O. P., \& Cerqueira, M. B. (2008). Saúde mental infanto-juvenil: Usuários e suas trajetórias de acesso aos serviços de saúde. Revista Espaço para a Saúde, 10(1), 3446. Recuperado em http://amazonia.fiocruz. br/arquivos/category/83-2013-09-17-0920-37? download=1688:2014-02-13-09-00$18 \&$ start $=60$

Fleitlich, B. A. (2005). Saúde nos jovens brasileiros: Quais são os principais problemas e como podemos ajudar? Jornal Brasileiro de Psiquiatria, 54(3), 168-169. doi:10.1590/S180637132004000800002

Guerra, A. M. C. (2003). Tecendo a rede na assistência em Saúde Mental Infanto-Juvenil: Interfaces entre a dimensão clínica e a dimensão política. In A. M. C. Guerra \& N. L. Lima (Eds.), Clínica de crianças com transtornos de desenvolvimen- to: Uma contribuição no campo da Psicanálise e da Saúde Mental (pp. 171-189). Belo Horizonte, MG: Autêntica.

Hoffmann, M. C. C. L., Santos, D. N., \& Mota, E. L. A. (2008). Caracterização dos usuários e dos serviços prestados por Centros de Atenção Psicossocial Infanto-Juvenil. Caderno de Saúde Pública, 24(3), 633-642. doi:10.1590/S0102$-311 X 2008000300017$

Mângia, E. F., Assumpção, C. N., Quinta, J. M., \& Rufino, M. de F. (2003). Necessidades de adolescentes com sofrimento psíquico. Revista de Terapia Ocupacional da USP, 14(3), 123-132. doi:10.11606/issn.2238-6149.v14i3p123-132

Matsukura, T. S., Fernandes, A. D. S. A., \& Cid, M. F. B. (2012). Fatores de risco e proteção à saúde mental infantil: O contexto familiar. Revista de Terapia Ocupacional da USP, 23(2), 122-129. doi:10.11606/issn.2238-6149.v23i2p122-129

Ministério da Saúde. (2011). Portaria $N^{o} 3.088$, de 23 de dezembro de 2011. Rede de atenção psicssocial. Recuperado em http://bvsms.saude.gov.br/ bvs/saudelegis/gm/2011/prt3088_23_12_2011_ rep.htm

Ministério da Saúde. (2012). Mais sobre os serviços disponíveis em Saúde Mental. Recuperado em http://portalsaude.saude.gov.br/index.php/cidadao/acoes-e-programas/conte-com-a-gente/leia-mais-conte-com-a-agente

Ministério da Saúde. (2014). Atenção psicossocial a crianças e adolescentes no SUS: Tecendo redes para garantir direitos. Brasília, DF: Autor.

Moraes, N. A., Dias, H. Z. J., Romio, C. M., \& Peixoto, M. J. R. (2011). Intervenção em saúde mental: Construindo um ambiente terapêutico em um Caps Infanto-Juvenil. Revista Contexto \& Saúde (Ijuí), 10(20). Recuperado em https:// www.revistas.unijui.edu.br/index.php/contextoesaude/article/view/1731

Navarini, V., \& Hirdes, A. (2008). A família do portador de transtorno mental: Identificando recursos adaptativos. Texto \& Contexto Enfermagem, 17(4), 680-688. doi:10.1590/S010407072008000400008

Oliveira, M. F. A. P. B. O. (2010). Trabalho oculto na rede de atenção psicossocial: As crianças $e$ jovens em sofrimento psíquico (Dissertação de mestrado, Faculdade de Saúde Publica, Universidade de São Paulo, SP, Brasil). Recuperado em http://bvsalud.org/portal/resource/pt/lil-594098 
Paludo, S., \& Koller, S. H. (2011). Inserção Ecológica no espaço da rua. In S. H. Koller (Ed.), Ecologia do desenvolvimento humano: Pesquisa e intervenção no Brasil. São Paulo, SP: Casa do Psicólogo.

Paula, C. S., Vedovato, M. S., Bordin, I. A S., Barros, M. G. S. M., D’Antino, M. E. F., \& Mercadante, M. T. (2008). Mental Health and violence among sixth grade students from a city I the state of São Paulo. Revista de Saúde Pública, 42(3), $524-$ 528. doi:10.1590/S0034-89102008005000027

Ronchi, J., \& Avellar, L. (2013). Ambiência na Atenção Psicossocial Infanto-Juvenil: Um estudo no CAPSi. Saúde e Sociedade, 22(4), 1045-1058. doi:10.1590/sausoc.v22i4.76499

Rutter, M. (1987). Psychosocial resilience and protective mechanisms. American Journal of Orthopsychiatry, 57(3), 316-331.

Santos, P. L. (2006). Problemas de saúde mental de crianças e adolescentes atendidos em um serviço público de psicologia infantil. Psicologia em Estudo, 11(2), 315-321. doi:10.1590/S141373722006000200010
Taño, B. L. (2014). Os Centros de Atenção Psicossocial Infantojuvenis (CAPSi) e as práticas de cuidado para as crianças e adolescentes em intenso sofrimento psíquico (Dissertação de mestrado, Programa de Pós-Graduação em Terapia Ocupacional, Universidade Federal de São Carlos, SP, Brasil).

Yin, R. K. (1994). Case study research: Design and methods. London: Sage.

Recebido: $28 / 01 / 2015$

$1{ }^{a}$ revisão: 06/08/2015

Aceite final: 27/08/2015 